\title{
Distribution of muscle weakness of central and peripheral origin
}

\author{
R D Thijs, N C Notermans, J H J Wokke, Y van der Graaf, J van Gijn
}

\begin{abstract}
According to the established clinical tradition about the distribution of weakness, the ratios of flexor/extensor strength of patients with upper motor neuron lesions are expected to be relatively high for the elbow and wrist and low for the knee. To assess the diagnostic value of these patterns of weakness, muscle strength of 70 patients with limb weakness of central or peripheral origin was measured with a hand held dynamometer. The ratios of flexor/extensor strength at the knee, elbow, and wrist did not differ significantly between patients with central or peripheral origin of muscle weakness. The examination of tendon jerks proved to be of more value as a localising feature. The traditional notion about the distribution of weakness in upper motor neuron lesions may be explained by an intrinsically greater strength in antigravity muscles, together with the effects of hypertonia. ( $($ Neurol Neurosurg Psychiatry 1998;65:794-796)
\end{abstract}

Keywords: muscle strength; dynamometry

Lesion localisation is a principal strategy in the evaluation of a patient with muscle weakness. Neurologists distinguish weakness of central and peripheral origin by means of muscle tone, fasciculations, reflexes, and additionally the distribution of weakness. ${ }^{1}$ Distinction between weakness of central and peripheral origin by means of the distribution of that weakness is a possible means of differentiation, although not as reliable as muscle tone, reflexes, and fasciculations. Central lesions are thought to affect extensors more than flexors in the upper limb and flexors more than extensors in the lower limb. ${ }^{1-3}$ Such a classic distribution of a central lesion is often referred to as a "pyramidal" pattern. This concept has been derived from careful clinical observations. ${ }^{3}$

Nevertheless, Colebatch and Gandevia ${ }^{4}$ found that flexors and extensors in the upper limb were equally affected in upper motor neuron lesions. Muscle strength of flexors and extensors of hemiparetic and hemiplegic patients was expressed as a fraction of the corresponding muscles on the unaffected side. In a similar study of the lower limb, flexors and extensors in hemiparetic and paraparetic patients also seemed to be equally affected. ${ }^{5}$ In both studies fixed dynamometers were used to measure muscle strength.

As no direct comparison has been made between patients with weakness of central and peripheral origin, the question remains whether a distinction on clinical grounds is accurate. To assess the diagnostic value of the distribution of weakness, we measured muscle strength in patients with limb weakness of central or peripheral origin, using a hand held dynamometer. In addition, we compared the weakness of proximal and distal muscle groups, another characteristic with potential value as a localising feature.

\section{Methods}

PATIENTS

Seventy patients were included in the study. All had been referred to the Department of Neurology of the University Hospital, Utrecht. For patients to be included the strength of at least one muscle group had to be grade 4 on the Medical Research Council (MRC) scale and the cause of the weakness should have been determined on other grounds as either of central or peripheral origin. Of the 31 patients with weakness of central origin, 10 patients had a cerebrovascular lesion, 10 had multiple sclerosis, six had spinal cord lesions, four had a cerebral tumour, and one had a head injury. Of 39 patients with weakness of peripheral origin, 21 had a polyneuropathy, three had spinal muscular atrophy, three had lumbar radiculopathy, and 12 had a myopathy.

Patients with pain or fatigue (for example, due to rheumatoid arthritis or myasthenia gravis) and patients with both central and peripheral impairment were excluded from the study.

\section{MEASUREMENTS}

Muscle strength of flexors and extensors of the hip, knee, ankle, elbow, and wrist was assessed on both sides with the MRC scale. ${ }^{6}$ In addition, these muscle groups were measured under similar conditions with a hand held dynamometer. Measurements of hip flexors, wrist extensors, and the flexors and extensors at the knee, ankle, and elbow were performed according to the methods of van der Ploeg et al. ${ }^{7}$ Hip extensors were measured with the dynamometer 
placed on the posterior surface of the distal thigh, and wrist flexors with a supinated forearm, the dynamometer being placed on the middle phalanges of the flexed fingers.

Each patient was tested once by the same examiner (RDT), who was not blinded to the diagnosis. Every muscle group was measured three times. Patients were instructed to increase their effort to a maximum for a period of about 3 seconds. The maximum of three readings was used as a measure of maximum voluntary contraction (MVC).

To permit comparison between subjects, flexor and extensor strength was expressed as a ratio by dividing the flexor's MVC by the MVC of the corresponding extensor.

Forces $>300 \mathrm{~N}$ were not included in the analysis, because such forces are not appropriate for this technique. ${ }^{8}$ Nearly all measurements of the hip extensors and foot plantar flexors had to be excluded for this reason (the patient's contraction could not be overcome by the examiner). As a consequence, ratios of flexor and extensor strength at the hip and ankle could not be obtained. All readings of the muscle groups of the knee, elbow, and wrist were well below $300 \mathrm{~N}$.

If a patient complained of pain or cramp during a test, the test was stopped and the reading was not included. Ten of 420 tests could not be completed for this reason. The knee jerks, ankle jerks, and the plantar responses were recorded. The tendon reflexes were graded on a 5 point scale (absent, diminished, normal, increased, clonus) and the plantar response was assessed as extensor, flexor, or mute.

If a proximal muscle group was rated as the lowest MRC grade within a limb, weakness was defined as proximal. If a distal muscle group was rated as the lowest MRC grade within a limb, weakness was defined as distal.

\section{ANALYSIS}

The ratio between flexor and extensor strength of both patient groups was compared by means of the Wilcoxon signed rank test or the MannWhitney $U$ test. $^{9}$ Regression analysis was used to evaluate the influence of age on the flexor/extensor ratio. The effect of sex on the ratio was tested with the Mann-Whitney $U$ test. Statistical significance was set at the 5\% level.

The variability of three readings was expressed as the coefficient of variation $(\mathrm{CV})$. The SD of three readings of one muscle group was divided by the mean of these readings (CV $(\%)=S D \times 100 /$ mean $)$. The CVs were calculated only if the MVC of a muscle group was used for the flexor/extensor ratio. CVs of several muscle groups were normalised by log transformations.

\section{Results}

Of the 70 patients, 45 were men. The median age of the patients was 49 (SD 14) years with a range of 17 to 77 years. The values of flexor and extensor strength and the median flexor/ extensor ratio at the knee, elbow, and wrist for patients with central and peripheral origin of muscle weakness are presented in the figure.
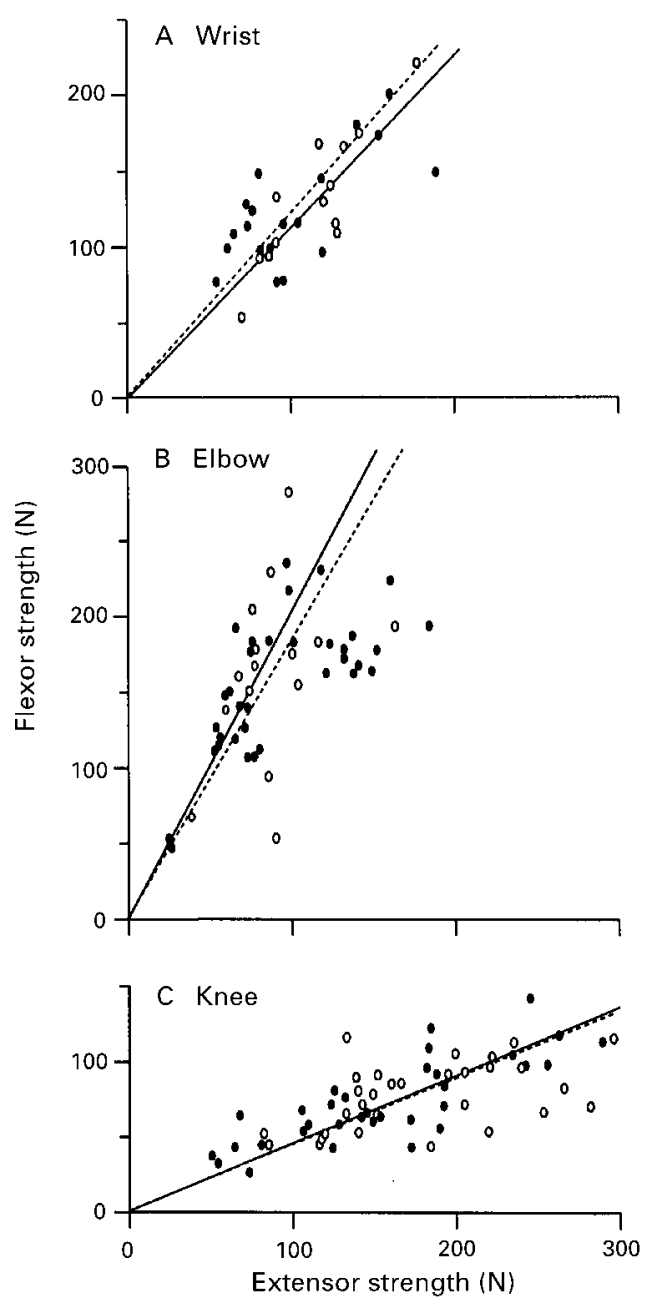

The absolute values of flexor and extensor muscle strength as measured with a hand held dynamometer at the knee, elbow, and wrist (left and right side combined) of patients with weakness of central (o) or peripheral $(\bullet)$ origin are shown. The gradient of the line is the median flexor/extensor ratio of patients with weakness of central (-) or peripheral (- - ) origin.

The plots demonstrate a similar distribution of flexor and extensor strength and the flexor/ extensor ratio for the central and peripheral group. For all muscle groups, the ratios for both groups of patients did not differ significantly. In the table the number of patients for each test, the median values of flexor and extensor strength, and the flexor/extensor ratio are given.

Regression analysis showed no significant effect of age on the flexor/extensor ratios. Similarly, no significant influence of sex on the ratios was seen.

The mean CV of three readings was $5.2 \%$, with a range of $3.3-7.3 \%$. All abnormal reflex patterns were in agreement with the location of the lesion. Nine patients had normal reflexes.

Of the patients with a muscle disease $92 \%$ had proximal weakness. Distal weakness in the upper or lower limbs was found in $52 \%$ of the patients with lesions of the central and $70 \%$ of the patients with lesions of the peripheral nervous system. 
Muscle strength and flexor/extensor ratios in 70 patients with muscle weakness of central or peripheral origin, measured with a hand held dynamometer

\begin{tabular}{|c|c|c|c|c|c|c|c|c|c|}
\hline & \multicolumn{4}{|c|}{ Central $(n=31)$} & \multicolumn{4}{|c|}{ Peripheral $(n=39)$} & \multirow[b]{2}{*}{$p$ Value * } \\
\hline & $n$ & Flex & Ext & Ratio (SD) & $n$ & Flex & Ext & Ratio (SD) & \\
\hline \multicolumn{10}{|l|}{ Proximal: } \\
\hline Left knee & 16 & 81 & 166 & $0.46(0.09)$ & 16 & 69 & 152 & $0.45(0.10)$ & 0.27 \\
\hline Right knee & 14 & 78 & 170 & $0.47(0.17)$ & 17 & 64 & 152 & $0.49(0.16)$ & 0.71 \\
\hline Left elbow & 7 & 177 & 85 & $1.81(0.73)$ & 17 & 169 & 96 & $1.81(0.44)$ & 0.62 \\
\hline Right elbow & 8 & 162 & 80 & $2.18(0.67)$ & 17 & 164 & 74 & $2.12(0.57)$ & 0.89 \\
\hline \multicolumn{10}{|l|}{ Distal: } \\
\hline Left wrist & 8 & 125 & 127 & $1.12(0.14)$ & 9 & 117 & 96 & $1.22(0.33)$ & 0.61 \\
\hline Right wrist & 5 & 135 & 92 & $1.25(0.27)$ & 10 & 123 & 86 & $1.25(0.33)$ & 0.44 \\
\hline
\end{tabular}

${ }^{\star} \mathrm{p}$ Value between the flexor/extensor ratio for patients with weakness of central and peripheral origin (Mann-Whitney $U$ test, two tailed).

Median values are given. Muscle strength is expressed in Newtons $(\mathrm{N}) . \mathrm{n}=$ number of patients, flex=flexor, ext=extensor; ratio $=$ flexor/extensor ratio

\section{Discussion}

According to the established clinical tradition about patterns of weakness, the ratios of flexor/ extensor strength of patients with upper motor neuron lesions are expected to be higher for the elbow and wrist and lower for the knee. We found no such difference. The distribution of weakness, at least as measured with a hand held dynamometer, has no localising value in terms of central or peripheral origin of weakness. Examination of myotatic reflexes combined with the distinction between proximal and distal weakness is clinically of more value as a localising feature.

The hand held dynamometer is a more objective method of strength assessment than the widely used grading scale of the MRC. ${ }^{10}$ This technique of muscle testing has been proved to be acceptable and reproducible. ${ }^{10}$ Readings of single raters, irrespective of experience, have a similar reproducibility and variability. ${ }^{11}$ The CVs of the readings from our study are in agreement with the findings of Wiles et $a l^{\beta}$ and Goonetilleke et al. ${ }^{11}$

Clinical assessment of muscle strength seems to be poorly differentiated, as an elbow flexor graded 4 on the MRC scale can, for example, vary between $10 \mathrm{~N}$ and $250 \mathrm{~N}$ on the scale of the dynamometer. ${ }^{10}$ Moreover, the MRC scale is ordinal and therefore cannot express the flexor strength as a fraction of the extensor force. In this study we did quantify the manual testing of flexor and extensor strength and our results did not confirm the clinical impression of the distribution of weakness of patients with lesions of the central or peripheral nervous system.

In a recent study of the clinical range of limb girdle muscular dystrophy the summation of paretic muscles showed a pattern of weakness similar to that in upper motor neuron lesions. ${ }^{12}$ Muscles particularly involved in the "pyramidal" distribution, are intrinsically weaker. ${ }^{457}$ These muscles are thereby predisposed to appear weak and this distribution is not particularly a central one. Nevertheless, we have to make the qualification that we could not calculate ratios for hip and foot muscles.
A distinct feature of upper motor neuron lesions is the increase of muscle tone. Tone has been shown to be independent of weakness. ${ }^{13}$ Although in clinical examination the terms hypotonia and normal tone seem to be inappropriate, because voluntary activity is responsible for most of the resistance felt ${ }^{14}$ the clinical examination of muscle tone is of great value for the detection of spasticity. Hypertonia tends to predominate in the flexors of the arm and the extensors of the leg. ${ }^{15}$ These muscles are intrinsically stronger. The traditional notion about the distribution of weakness in upper motor neuron lesions may be explained by a combination of two factors: an intrinsic difference in strength (flexors being stronger in the arms and extensors in the legs), and the occurrence of hypertonia in the stronger muscle groups.

1 Lindsay KW, Bone I, Callander R. Neurology and neurosurgery illustrated. Edinburgh: Churchill Livingstone, 1991:189.

2 Lance JW, McLeod JG. A physiological approach to clinical neurology. London: Butterworth, 1981:30-45.

3 Wernicke C. Zur Kenntniss der cerebralen Hemiplegie. Berliner Klinische Wochenschrift 1889;26:969-70.

4 Colebatch JG, Gandevia SC. The distribution of muscular weakness in upper motor neuron lesions affecting the arm. Brain 1989;112:749-63.

5 Adams RW, Gandevia SC, Skuse NF. The distribution of muscle weakness in upper motor neuron lesions affecting the lower limb. Brain 1990;113:1459-76.

6 Medical Research Council. Aids to the investigation of the peripheral nervous system. London: Her Majesty's Stationery Office, 1975:1-2.

7 Ploeg RJO van der, Fidler V, Oosterhuis HJGH. Hand-held myometry: reference values. $\mathcal{F}$ Neurol Neurosurg Psychiatry 1991;54:244-7

8 Wiles CM, Karni Y. The measurement of muscle strength in patients with peripheral neuromuscular disorders. $\mathcal{f}$ Neurol Neurosurg Psychiatry 1983;46:1006-13.

9 Mann HB, Whitney DR. On a test of whether one of two random variables is stochastically larger than the other. Annals of Mathematical Statistics 1947;18:50-60.

10 Ploeg RJO van der, Oosterhuis HJGH, Reuvekamp J. Measuring muscle strength. F Neurol 1984;231:200-3.

11 Goonetilleke A, Modarres-Sadeghi H, Guiloff RJ. Accuracy, reproducibility, and variability of hand-held dynamometry in motor neuron disease. F Neurol Neurosurg Psychiatry 1994;57:326-32

12 Kooi AJ van der, Barth PG, Busch HFM, et al. The clinical spectrum of limb girdle muscular dystrophy: a survey in the Netherlands. Brain 1996;119:1471-80.

13 O'Dwyer NJ, Ada L, Neilson PD. Spasticity and muscle contracture following stroke. Brain 1996;119:1737-49.

14 Meché FGA van der, Gijn J van. Hypotonia: an erroneous clinical concept? Brain 1986;109:1169-78.

15 Walton J. Brain's diseases of the nervous system. Oxford: Oxford University Press, 1985:33. 